\title{
EFICÁCIA TERAPÊUTICA DO PROGRAMA DE REMEDIAÇÃO FONOLÓGICA EM ESCOLARES COM DISLEXIA DO DESENVOLVIMENTO
}

\section{Therapeutic effectiveness of phonological remediation program in students with developmental dyslexia}

\author{
Simone Aparecida Capellini ${ }^{(1)}$, Maria Nobre Sampaio (2), \\ Kelssy Hitomi dos Santos Kawata ${ }^{(3)}$, Niura Aparecida de Moura Ribeiro Padula (4), \\ Lara Cristina Antunes dos Santos ${ }^{(5)}$, Maria Dalva Lorencetti ${ }^{(6)}$, lan Smythe ${ }^{(7)}$
}

\begin{abstract}
RESUMO
Objetivos: comparar os achados da avaliação em situação de pré e pós-testagem em escolares com dislexia do desenvolvimento e escolares bons leitores submetidos ao programa de remediação fonológica e verificar a eficácia terapêutica do programa de remediação fonológica em escolares com dislexia do desenvolvimento. Métodos: participaram deste estudo 40 escolares de $2^{a}$ a $4^{a}$ série de ensino público do município de Marília-SP, de ambos os sexos, na faixa etária de 8 a 12 anos distribuídos em: Gl: composto de 20 escolares com diagnóstico interdisciplinar de dislexia do desenvolvimento que foram submetidos a programa de remediação fonológica, Gll: composto de 20 escolares sem dificuldades de aprendizagem da rede municipal de ensino público, pareados segundo sexo, faixa etária e escolaridade com os escolares do GI que não foram submetidos aos programas de remediação. Em situação de pré e pós-testagem, todos os escolares foram submetidos à aplicação do Teste de Desempenho Cognitivo-Linguístico nas versões coletiva e individual, seguido de leitura oral e compreensão de textos. Resultados: foram evidenciadas diferenças estatisticamente significantes, indicando que os escolares do Gl e Gll submetidos ao programa de remediação fonológica apresentaram desempenho superior em situação de pós-testagem em comparação com a situação de pré-testagem para a maioria das habilidades cognitivo-linguísticas avaliadas, incluindo a leitura e compreensão de texto. Conclusão: o programa de remediação fonológica para crianças com e sem dislexia do desenvolvimento foi eficaz, sugerindo que a habilidade de relação letra-som deve ser utilizada em contexto de sala de aula favorecendo a leitura desses escolares.
\end{abstract}

DESCRITORES: Dislexia; Leitura; Estudos de Intervenção

(1) Fonoaudióloga; Docente do Departamento de Fonoaudiologia e do Programa de Pós-Graduação em Educação da Faculdade de Filosofia e Ciências da Universidade Estadual Paulista, FFC-UNESP, Marília, SP; Doutora em Ciências Médicas pela Faculdade de Ciências Médicas da Universidade Estadual de Campinas.

(2) Fonoaudióloga voluntária do Departamento de Fonoaudiologia da Faculdade de Filosofia e Ciências da Universidade Estadual Paulista, FFC-UNESP, Marília, SP.

(3) Aluna do Curso de Fonoaudiologia da Faculdade de Filosofia e Ciências da Universidade Estadual Paulista, FFCUNESP, Marília, SP; Bolsista da Fundação de Amparo à Pesquisa do Estado de São Paulo sob Processo $n^{\circ}$ 07/51842-0.

(4) Neurologista Infantil; Docente do Departamento de Neurologia e Psiquiatria da Faculdade de Medicina de Botucatu da Universidade Estadual Paulista, UNESP, Botucatu, SP; Doutora em Ciências Médicas pela Faculdade de Ciências Médicas da Universidade Estadual de Campinas.

(5) Neurologista Infantil do Ambulatório de Desvios da Aprendizagem do Hospital das Clínicas da Faculdade de Medicina de Botucatu da Universidade Estadual Paulista, UNESP, Botucatu, SP.

(6) Neuropsicóloga do Ambulatório de Desvios da Aprendizagem do Hospital das Clínicas da Faculdade de Medicina de Botucatu da Universidade Estadual Paulista, UNESP, Botucatu, SP.

(7) Engenheiro; Professor visitante da Universidade Normal do Sul da China, Guangzhou, China; Doutor em Psicologia Cognitiva pela University of Surrey.

Conflito de interesses: inexistente 


\section{INTRODUÇÃO}

A dislexia do desenvolvimento é uma dificuldade de aquisição de leitura e escrita, apesar da preservação da inteligência, oportunidade de aprendizagem, motivação e acuidade sensorial ${ }^{1,2}$. A dificuldade central da dislexia está no componente fonológico, o qual é responsável por acessar as estruturas sonoras subjacentes às palavras. O problema do escolar com dislexia do desenvolvimento tem sua origem durante o desenvolvimento do cérebro, com a presença de más formações corticais e subcorticais originadas no período embrionário. Essas más formações ocorrem em áreas vinculadas ao processamento fonológico, como nas áreas de Broca e de Wernicke, regiões de produção e compreensão da linguagem oral e escrita ${ }^{2}$.

O déficit fonológico presente na dislexia interfere na aprendizagem da correspondência letra-som, necessária para a aquisição da leitura. Os disléxicos apresentam dificuldades na percepção dos fonemas necessários para organizar as representações ortográficas de um sistema de escrita com base alfabética ${ }^{3}$.

Em decorrência desse déficit, pesquisas realizadas desde a década de 80 descrevem a necessidade da realização de programas de intervenção, também conhecidos internacionalmente como programas de remediação, que enfatizem o ensino da relação letra-som e das habilidades metalinguísticas necessárias para a aprendizagem do sistema de escrita com base alfabética. Estudos internacionais ${ }^{4-7}$ desenvolveram programas de remediação para o tratamento das dificuldades de leitura por meio do treinamento da consciência fonológica e ensino explícito das regras de correspondência grafofonêmicas.

Esses estudos descreveram o uso de habilidades metafonológicas para o desenvolvimento da leitura e escrita. Os programas são geralmente baseados em atividades fonêmicas, silábicas e suprafonêmicas (rima e aliteração), que têm como objetivo desenvolver habilidades referentes ao processamento fonológico relacionado com a leitura e a compreensão textual (velocidade de acesso ao léxico, nomeação e consciência fonológica). As atividades presentes nesses programas de treinamento fonológico incluem o seguinte esquema: estrutura silábica da palavra (análise e síntese); identificação de sílabas; identificação de fonemas; comparação de sílabas; comparação de fonemas; recombinação silábica (segmentação e manipulação); recombinação fonêmica (segmentação e manipulação) e identificação de sons e sílabas por rima e aliteração ${ }^{4-7}$.

No Brasil, os programas de remediação também tiveram o seu início na década de 80 , com pesquisas que se propunham a realizar programas de remediação com a leitura para a melhoria da compreensão de textos em crianças do ensino básico. Entre essas pesquisas destaca-se o uso de procedimentos metodológicos de pré-testagem, treinamento com leitura em 15 sessões e pós-testagem, com o objetivo de verificar a eficácia do uso da técnica de cloze ou instrução programada para o ensino da leitura ${ }^{8}$.

Os programas direcionados para o ensino da instrução direta da consciência fonológica juntamente com a instrução da correspondência fonema-grafema para a aquisição do princípio alfabético do sistema de escrita do português tiveram seu início no Brasil 9-11. De modo geral, essas pesquisas visaram verificar a influência da consciência fonológica para o desenvolvimento da leitura e escrita. Apesar de na literatura internacional serem vastos os estudos que descrevem o uso de programas de remediação fonológica com crianças que apresentam dislexia do desenvolvimento, no Brasil, esses estudos ainda são restritos ${ }^{12-14}$, evidenciando a necessidade na continuidade de pesquisas que adaptem programas de remediação utilizados internacionalmente para a realidade brasileira ou que elaborem procedimentos de remediação baseados no sistema de escrita do português.

Com base no exposto acima, esse estudo teve como objetivos comparar os achados dos procedimentos de avaliação utilizados na pré e póstestagem em escolares com dislexia do desenvolvimento e escolares sem dificuldades submetidos ao programa de remediação fonológica, bem como verificar a eficácia terapêutica do programa de remediação fonológica em escolares com dislexia do desenvolvimento.

\section{MÉTODOS}

Esta pesquisa é longitudinal retrospectiva e visa apresentar o estudo de escolares disléxicos e controles submetidos a um programa de remediação fonológica.

Participaram desse estudo 40 escolares de $2^{2}$ a 4⿳亠丷a série do Ensino Público Fundamental, de ambos os sexos, com idade entre 8 a 12 anos, distribuídos em dois grupos:

- Grupo I (GI): grupo composto de 20 escolares com o diagnóstico interdisciplinar de dislexia do desenvolvimento, sendo 16 (80\%) do sexo masculino e $4(20 \%)$ do feminino, com faixa etária de 9 a 12 anos, com média etária de 10 anos e 3 meses, sendo que $4(20 \%)$ dessa população estavam na $2^{\mathrm{a}}$ série, $4(20 \%)$ na $3^{\text {a }}$ série e 12 $(60 \%)$ na $4^{a}$ série, subdivididos em: 
- Grupo le (Gle): composto de 10 escolares com dislexia do desenvolvimento, submetidos ao programa de remediação fonológica.

- Grupo Ic (GIc): composto de 10 escolares com dislexia do desenvolvimento, que não foram submetidos ao programa de remediação fonológica.

- Grupo II (GII): grupo composto de 20 escolares, de ambos os sexos, com faixa etária de 9 a 12 anos, com média etária de 10 anos e 3 meses, pareados segundo gênero, escolaridade e faixa etária com o Gl, subdivididos em:

- Grupo lle (Glle): composto de 10 escolares com bom desempenho escolar submetidos ao programa de remediação fonológica;

- Grupo IIc (GIIc): composto de 10 escolares com bom desempenho escolar que não foram submetidos ao programa de remediação fonológica.

O GI foi composto de escolares encaminhados ao Centro de Estudos da Educação e da Saúde - CEES, da Universidade Estadual Paulista UNESP, Campus de Marília - SP, com queixa de dificuldades de aprendizagem. Esses escolares foram submetidos à avaliação fonoaudiológica no Centro de Estudos da Educação e Saúde - CEES/ FFC/UNESP-Marília-SP, e, depois da verificação dos sinais da dislexia, ou seja, presença de transtorno fonológica na fala, leitura e escrita, alterações em habilidades fonológicas e silábicas, presença de histórico familial positivo para dislexia, esses escolares foram encaminhados para avaliação neurológica, neuropsicológica e de imagem no Ambulatório de Neurologia Infantil - Desvios da Aprendizagem do Hospital das Clínicas da Faculdade de Medicina de Botucatu da UNESP, tendo sido confirmado o diagnóstico de dislexia do desenvolvimento.

Os escolares do GI foram considerados disléxicos quando apresentaram os seguintes critérios em situação de avaliação interdisciplinar: alteração quanto ao equilíbrio estático, à coordenação apendicular, persistência motora, equilíbrio dinâmico, coordenação tronco-membro e sensibilidade no exame neurológico evolutivo, discrepância entre coeficiente intelectual verbal e execução na avaliação psicológica, alteração quanto à memória, leitura e escrita na bateria neuropsicológica, alterações fonêmicas, silábicas, rima e aliteração em provas de consciência fonológica, nível de leitura alfabético, velocidade de leitura oral abaixo do esperado para idade e escolaridade, transtorno fonológico evidenciados na avaliação fonológica, na leitura oral de textos e na leitura oral de palavras isoladas e na escrita sob ditado de palavras e pseudopalavras e na redação temática, compreensão parcial do texto lido.

O Gll foi constituído de escolares com bom desempenho escolar, ou seja, escolares que obti- veram desempenho satisfatório em dois bimestres consecutivos em avaliações de Língua Portuguesa indicados pelas professoras de $2^{\mathrm{a}}$ a $4^{\mathrm{a}}$ série do ensino público fundamental do Município de Marília-SP.

Neste estudo, foi realizada a adaptação brasileira de pesquisa desenvolvida a partir de remediação fonológica ${ }^{15}$.

Em situação de pré e pós-testagem, todos os escolares foram submetidos aos mesmos procedimentos para verificação de eficácia terapêutica dos programas utilizados. A escolha dos procedimentos de pré e pós testagem seguiu os mesmos critérios da avaliação utilizada na pesquisa dos autores citados, ou seja, instrumentos que verificassem a leitura de palavras e não-palavras isoladas, habilidade fonológica (rima, aliteração), leitura e compreensão de texto e escrita sob ditado de palavras isoladas.

Para a realização deste estudo, foram utilizados os seguintes procedimentos em situação de pré e pós-testagem:

A) Termo de consentimento Pós-Informado: Conforme resolução do Conselho Nacional de Saúde CNS 196/96, anteriormente ao início das avaliações, os pais ou responsáveis pelos escolares selecionados assinaram o termo de Consentimento Pós-Informado para autorização da realização do estudo.

B) Teste de Desempenho Cognitivo - Linguístico - versão coletiva: Foi aplicada a versão coletiva do Teste adaptada para o Brasil ${ }^{16,17} \mathrm{em}$ todos os escolares simultaneamente. Essa versão é composta de cinco subtestes:

- Reconhecimento do alfabeto em sequência: o escolar deveria escrever o alfabeto em sequência;

- Cópia de formas: o escolar deveria copiar quatro diferentes formas geométricas;

- Aritmética: o escolar deveria solucionar 20 operações aritméticas simples, que incluíam adição, subtração, multiplicação e divisão;

- Ditado: o escolar deveria escrever 30 palavras reais e 10 palavras inventadas;

- Memória de curta duração: o escolar deveria escrever 16 sequências de dígitos, que podem conter de dois a nove dígitos.

Os resultados dos subtestes da versão coletiva são apresentados em forma de pontuação, sendo atribuído 1 ponto a cada acerto do escolar. Além da pontuação, o desempenho de cada escolar foi analisado quanto à qualidade da escrita, sendo atribuído escore de 1 a 5 para a qualidade da escrita, e, quanto ao aparecimento de espelhamento, em que foi contado o número total de espelhamentos ocorridos no subteste de memória de curta duração. 
C) Teste de Desempenho Cognitivo - Linguístico - versão individual: Foi aplicada versão individual adaptada para o Brasil ${ }^{16,17}$. Esta versão é composta de 10 subtestes.

1) Leitura de palavras e não-palavras: composta de subtestes de leitura de palavras, leitura de palavras corretas em 1 minuto e leitura de não-palavras;

2) Consciência fonológica: composta de subtestes de aliteração e rima;

3) Habilidade matemática: composta de subtestes de aritmética e contagem decrescente;

4) Processamento auditivo: composta de subtestes de discriminação de sons, repetição de números, repetição inversa de números, batidas rítmicas, repetição de palavras e repetição de não-palavras;

5) Escrita: composta de subtestes de ditado de palavras, ditado de não-palavras, ditado total, primeiras letras no ditado e qualidade da escrita;

6) Processamento visual: composta de subtestes de memória visual para formas;

7) Velocidade de processamento: composta de subtestes de nomeação rápida de figuras e nomeação rápida de números;

8) Sequenciamento: composta de subtestes de alfabeto e sequências;

9) Habilidade motora: composta de subtestes de coordenação motora de mão direita, esquerda e ambas as mãos;

10) Reversões: composta de subtestes de reversões de números.

Os resultados dos subtestes da versão individual foram obtidos por meio de pontuação que apresentou variação entre 1 a 40 pontos.

D) Leitura Oral e Compreensão de Texto: Foram utilizados três textos, selecionados a partir da indicação de 14 professores de $2^{-}$a $4^{\text {a }}$ série da Rede Municipal de Ensino de Marília - SP. Os professores foram orientados a escolher um texto para cada série escolar dentre três opções de texto. Os textos com maior índice de aprovação por parte dos professores foram selecionados para realização da leitura oral e compreensão de textos. Este procedimento foi aplicado pelos pesquisadores.

A compreensão do texto foi realizada por meio de quatro perguntas apresentadas sequencialmente ao texto, em que os escolares deveriam escrever as respostas.

Os textos e as perguntas referentes aos textos foram apresentados aos escolares em papel A4, digitado em letra Arial, tamanho 16, cor preta, espaço duplo. Cada escolar teve o seu texto para leitura e compreensão.
A análise da leitura dos textos foi realizada a partir da contagem dos erros das palavras lidas (exatidão de leitura), tempo total de leitura e velocidade de leitura, e medição de palavras por minuto 18,19. O número de palavras por minuto foi calculado mutiplicando-se o número de palavras do texto por sessenta (60 segundos) e esse valor dividido pelo tempo total da leitura em segundos, conforme a seguinte fórmula:

Velocidade de leitura $=\underline{\text { número de palavras do texto } X 60}$ tempo total de leitura do texto

As questões dos textos foram analisadas para compreensão a partir da contagem das respostas corretas relacionadas com perguntas sobre o texto, sendo 4 respostas corretas $=100 \%$ de acerto $=$ compreensão total, 3 respostas corretas $(75 \%), 2$ respostas corretas (50\%), 1 resposta correta (25\%) = compreensão parcial, nenhuma resposta correta = sem compreensão de leitura.

O programa de remediação fonológica utilizado propõe o treinamento fonológico a partir de dez etapas de trabalho sistemático, a saber:

1) Identificação do som e da letra: foram apresentadas em folha de papel A4 todas as letras do alfabeto e os escolares nomeavam as letras e identificavam os sons do alfabeto. A análise das respostas foi calculada por pontos atribuídos para o acerto do som das letras.

2) Identificação de palavras dentro de uma frase: foram apresentadas 7 frases afirmativas auditivamente para os escolares dividirem a frase em palavras, marcando-as por palmas.

3) Identificação e manipulação de sílabas na palavra: foram apresentadas 2 palavras auditivamente para identificação de sílabas iguais e logo depois, foi solicitado ao escolar a manipulação de segmentação silábica para formação de novas palavras em posição inicial, medial e final. Em cada sessão foram fornecidas 6 palavras;

4) Síntese fonêmica: foram apresentadas 7 palavras auditivamente separadas por sons, e os escolares deveriam reconhecer as palavras.

5) Rima: foi solicitado aos escolares que dissessem palavras que terminassem com o mesmo som e identificassem figuras que rimassem em sua nomeação.

6) Identificação e discriminação de fonemas: foi apresentado auditivamente um fonema $e$ solicitado aos escolares que mencionassem uma palavra que começasse com este som. Depois, foram apresentados oralmente 7 palavras e perguntado se essas palavras tinham o fonema-alvo naquela palavra. Os fonemas foram apresentados seguindo a ordem de aquisição e desenvolvimento do sistema fonológico. 
7) Segmentação de fonemas: foi apresentado aos escolares 1 palavra oralmente e solicitado às crianças que dissessem todos os fonemas dessa palavra. Nessa fase do programa, foram utilizadas fichas coloridas para auxiliar os escolares a segmentar os sons das palavras auditivamente apresentadas. Em cada sessão foram fornecidas 7 palavras;

8) Subtração de fonemas: foram apresentadas 6 palavras para que o escolar retirasse o fonema final; e, em seguida, apresentadas 6 palavras para que o escolar retirasse o fonema inicial.

9) Substituição de fonemas: foi ditada 1 palavra e foi solicitado aos escolares que retirassem o fonema inicial e o substituísse por outro, formando, assim, uma nova palavra. Em cada sessão foram fornecidas 7 palavras;

10) Transposição de fonemas: foi solicitado aos escolares que falassem as palavras em ordem inversa da palavra apresentada para formar novas palavras.

As dez etapas do programa fonológico foram trabalhadas em 18 sessões individuais, realizadas uma vez por semana com 50 minutos de duração cada, portanto, o programa foi realizado em 4 meses e 2 semanas. Os professores dos escolares deste estudo foram orientados a utilizar aleatoriamente as estratégias do programa no contexto de sala de aula. Após este período de remediação foram aplicados os procedimentos de pós-testagem.

Esse estudo foi realizado depois da aprovação do Comitê de Ética em Pesquisa da Faculdade de Filosofia e Ciências da Universidade Estadual Paulista - CEP/FFC/UNESP, sob o protocolo número 3325/2006.

Os resultados desse estudo foram analisados estatisticamente visando comparar o desempenho dos escolares em situação de pré e pós-testagem e durante o programa de remediação. Para a análise estatística, foram utilizados: o Teste de Mann-Whitney, com o intuito de verificar possíveis diferenças entre os grupos; o Teste dos Postos Sinalizados de Wilcoxon, com o intuito de verificar possíveis diferenças de desempenho entre os dois momentos de testagem dos grupos; e o Teste de Friedman, para verificar possíveis diferenças entre as variáveis de componentes de cada etapa do programa de remediação fonológica.

Para a análise estatística, foi adotado o nível de significância de 5\% (a = 0,050 - significância adotada), ou seja, quando a significância calculada (p) fosse menor que $5 \%(0,050)$, então, havia uma diferença (ou relação) estatisticamente significante (marcada com asterisco); quando a significância calculada $(p)$ fosse igual ou maior que $5 \%(0,050)$, então, havia uma diferença (ou relação) estatisti- camente não significante, isto é, uma semelhança. A análise dos dados foi realizada com a utilização do programa SPSS (Statistical Package for Social Sciences), em sua versão 13.0.

\section{RESULTADOS}

As Tabelas 1 e 2 apresentam respectivamente a comparação do desempenho do Glc e Gllc em situação de pré e pós-testagem.

Com a aplicação do Teste dos Postos Sinalizados de Wilcoxon, pode-se verificar que tanto os escolares do Glc como do Gllc mantiveram os seus desempenhos nos dois momentos de testagem, ou seja, na pré e na pós-testagem.

As Tabelas 3 e 4 apresentam respectivamente a comparação do desempenho do Gle e Glle em situação de pré-testagem e pós-testagem.

Aplicando o Teste dos Postos Sinalizados de Wilcoxon, pode-se observar diferença estatisticamente significante tanto para o Gle como para o Glle quando foram comparados os dois momentos de testagem. Como mostra a Tabela 3, apenas não foi verificada diferença estatisticamente significante para o Gle nas habilidades de cópia de formas e contagem de números em ordem inversa. A Tabela 4 mostra que não foi verificada diferença estatisticamente significante para o Gll nas habilidades de leitura de palavras, repetição de pseudopalavras, memória visual para 4 e 5 formas, contagem de números em ordem inversa e sequência de dias da semana e meses do ano.

Estas tabelas mostram a eficácia do programa de remediação fonológica tanto para o grupo de escolares disléxicos como para o grupo de escolares sem problemas de aprendizagem.

A Tabela 5 apresenta a comparação do desempenho do Gl e Gll em situação de pré-testagem e pós-testagem para leitura e compreensão do texto lido.

Depois da aplicação do Teste dos Postos Sinalizados de Wilcoxon, pode-se observar que tanto os escolares do Gle como os escolares do Glle, que foram submetidas ao programa de remediação fonológica, apresentaram desempenho superior em situação de pós-testagem se comparado o desempenho na pré-testagem, pois ocorreu diferença estatisticamente significante, indicando diminuição do número de palavras lidas por minuto, diminuição do tempo total de leitura e compreensão do texto lido.

No que se refere ao Glc, não submetido ao programa de remediação fonológica, pôde-se observar diferença estatisticamente significante em compreensão do texto lido. 
Tabela 1 - Distribuição das médias, desvio-padrão e significância de $p$ do desempenho dos escolares do Glc em situação de pré-testagem e pós-testagem

\begin{tabular}{|c|c|c|c|c|}
\hline Par de Variáveis & $\mathrm{n}$ & Média & Desvio-padrão & Significância (p) \\
\hline Alf_Pré & 10 & 16,90 & 2,81 & \multirow{2}{*}{0,317} \\
\hline Alf_Pós & 10 & 17,00 & 2,75 & \\
\hline LP_Pré & 10 & 714,50 & 287,16 & \multirow{2}{*}{0,336} \\
\hline LP_Pós & 10 & 715,00 & 287,49 & \\
\hline LNP_Pré & 10 & 80,20 & 28,56 & \multirow{2}{*}{0,414} \\
\hline LNP_Pós & 10 & 81,40 & 30,46 & \\
\hline Cor1m_Pré & 10 & 9,80 & 5,83 & \multirow{2}{*}{$>0,999$} \\
\hline Cor1m_Pós & 10 & 9,80 & 5,83 & \\
\hline Dit_P_Pré & 10 & 6,20 & 2,97 & \multirow{2}{*}{$>0,999$} \\
\hline Dit_P_Pós & 10 & 6,20 & 2,97 & \\
\hline DitNP_Pré & 10 & 1,30 & 0,48 & \multirow{2}{*}{$>0,999$} \\
\hline DitNP_Pós & 10 & 1,30 & 0,48 & \\
\hline Alit_Pré & 10 & 7,10 & 1,37 & \multirow{2}{*}{$>0,999$} \\
\hline Alit_Pós & 10 & 7,10 & 1,37 & \\
\hline Rima_Pré & 10 & 12,10 & 4,18 & \multirow{2}{*}{$>0,999$} \\
\hline Rima_Pós & 10 & 12,10 & 4,18 & \\
\hline D_S_Pré & 10 & 2,80 & 1,23 & \multirow{2}{*}{$>0,999$} \\
\hline D_S_Pós & 10 & 2,80 & 1,23 & \\
\hline RepP_Pré & 10 & 2,00 & 0,82 & \multirow{2}{*}{$>0,999$} \\
\hline RepP_Pós & 10 & 2,00 & 0,82 & \\
\hline RepNP_Pré & 10 & 3,50 & 0,53 & \multirow{2}{*}{$>0,999$} \\
\hline RepNP_Pós & 10 & 3,50 & 0,53 & \\
\hline PC_Pré & 10 & 29,30 & 10,88 & \multirow{2}{*}{0,655} \\
\hline PC_Pós & 10 & 29,20 & 10,77 & \\
\hline MV2_Pré & 10 & 2,00 & 0,67 & \multirow{2}{*}{$>0,999$} \\
\hline MV2_Pós & 10 & 2,00 & 0,67 & \\
\hline MV3_Pré & 10 & 1,40 & 0,52 & \multirow{2}{*}{$>0,999$} \\
\hline MV3_Pós & 10 & 1,40 & 0,52 & \\
\hline MV4_Pré & 10 & 0,90 & 0,57 & \multirow{2}{*}{$>0,999$} \\
\hline MV4_Pós & 10 & 0,90 & 0,57 & \\
\hline MV5_Pré & 10 & 2,00 & 0,00 & \multirow{2}{*}{0,157} \\
\hline MV5_Pós & 10 & 1,80 & 0,42 & \\
\hline Nfig_T_Pré & 10 & 117,30 & 27,06 & \multirow{2}{*}{0,672} \\
\hline Nfig_T_Pós & 10 & 121,30 & 26,45 & \\
\hline NN_T_Pré & 10 & 139,40 & 19,56 & \multirow{2}{*}{$>0,999$} \\
\hline NN_T_Pós & 10 & 139,40 & 19,56 & \\
\hline CF_Pré & 10 & 5,10 & 2,28 & \multirow{2}{*}{$>0,999$} \\
\hline CF_Pós & 10 & 5,10 & 2,28 & \\
\hline Matem_Pré & 10 & 3,40 & 2,55 & \multirow{2}{*}{$>0,999$} \\
\hline Matem_Pós & 10 & 3,40 & 2,55 & \\
\hline Memor_Pré & 10 & 6,90 & 2,23 & \multirow{2}{*}{0,763} \\
\hline Memor_Pós & 10 & 7,00 & 1,63 & \\
\hline Contg_Pré & 10 & 96,90 & 43,04 & 070 \\
\hline Contg_Pós & 10 & 94,10 & 43,13 & 0,786 \\
\hline Ritmo_Pré & 10 & 2,70 & 0,82 & \\
\hline Ritmo_Pós & 10 & 2,70 & 0,67 & $>0,999$ \\
\hline M_I_Pré & 10 & 1,30 & 0,48 & ᄀ 990 \\
\hline M_I_Pós & 10 & 1,30 & 0,48 & $>0,999$ \\
\hline Seq_Pré & 10 & 0,70 & 0,48 & \\
\hline Seq_Pós & 10 & 1,00 & 0,00 & 0,083 \\
\hline
\end{tabular}

Legenda: Alf_: alfabeto; $C_{-}$: cópia de formas; Matem_: matemática; Dit_P_: ditado de palavras; DitNP_: ditado de não-palavras; Memor_: memória; Cor1m_: palavras lidas corretamente em 1 minuto; PC_: palavras lidas corretamente; LP_: leitura de palavras; LNP_: leitura de não-palavras; Alit_: aliteração; Contg_: contagem; RepP_: repetição de palavras; RepNP_: repetição de não-palavras; Nfig_T_: nomeação de figuras; NN_T_: nomeação de números; MV2_: memória visual duas sequências; MV3_: memória visual três sequências; MV4_: memória visual quatro sequências; MV5_: memória visual cinco sequências; D_S_: discriminação de sons; M_I_: memória indireta; Seq_: sequências. 
Tabela 2 - Distribuição das médias, desvio-padrão e significância de p do desempenho dos escolares do Gllc em situação de pré-testagem e pós-testagem

\begin{tabular}{|c|c|c|c|c|}
\hline Par de Variáveis & $n$ & Média & Desvio-padrão & Significância (p) \\
\hline Alf_Pré & 10 & 23,10 & 2,60 & \multirow{2}{*}{0,796} \\
\hline Alf_Pós & 10 & 22,60 & 4,65 & \\
\hline LP_Pré & 10 & 246,80 & 101,27 & \multirow{2}{*}{0,507} \\
\hline LP_Pós & 10 & 217,30 & 116,36 & \\
\hline LNP_Pré & 10 & 15,00 & 3,62 & \multirow{2}{*}{0,258} \\
\hline LNP_Pós & 10 & 13,00 & 4,92 & \\
\hline Cor1m_Pré & 10 & 25,30 & 10,79 & \multirow{2}{*}{0,210} \\
\hline Cor1m_Pós & 10 & 24,00 & 10,74 & \\
\hline Dit_P_Pré & 10 & 14,50 & 5,82 & \multirow{2}{*}{0,932} \\
\hline Dit_P_Pós & 10 & 14,50 & 6,70 & \\
\hline DitNP_Pré & 10 & 2,30 & 1,16 & \multirow{2}{*}{0,480} \\
\hline DitNP_Pós & 10 & 2,60 & 1,51 & \\
\hline Alit_Pré & 10 & 7,00 & 2,62 & \multirow{2}{*}{0,071} \\
\hline Alit_Pós & 10 & 7,80 & 2,20 & \\
\hline Rima_Pré & 10 & 11,60 & 6,15 & \multirow{2}{*}{0,797} \\
\hline Rima_Pós & 10 & 11,80 & 5,18 & \\
\hline D_S_Pré & 10 & 16,10 & 2,88 & \multirow{2}{*}{0,705} \\
\hline D_S_Pós & 10 & 16,20 & 3,16 & \\
\hline RepP_Pré & 10 & 2,90 & 0,99 & \multirow{2}{*}{0,414} \\
\hline RepP_Pós & 10 & 3,10 & 0,74 & \\
\hline RepNP_Pré & 10 & 4,10 & 0,74 & \multirow{2}{*}{0,564} \\
\hline RepNP_Pós & 10 & 4,00 & 0,82 & \\
\hline PC_Pré & 10 & 28,40 & 8,54 & \multirow{2}{*}{$>0,999$} \\
\hline PC_Pós & 10 & 28,40 & 8,54 & \\
\hline MV2_Pré & 10 & 3,20 & 1,32 & \multirow{2}{*}{0,317} \\
\hline MV2_Pós & 10 & 3,10 & 1,37 & \\
\hline MV3_Pré & 10 & 4,30 & 1,25 & \multirow{2}{*}{0,102} \\
\hline MV3_Pós & 10 & 3,90 & 1,45 & \\
\hline MV4_Pré & 10 & 5,50 & 1,58 & \multirow{2}{*}{0,763} \\
\hline MV4_Pós & 10 & 5,40 & 2,17 & \\
\hline MV5_Pré & 10 & 6,00 & 1,94 & \multirow{2}{*}{0,589} \\
\hline MV5_Pós & 10 & 6,20 & 2,39 & \\
\hline Nfig_T_Pré & 10 & 44,30 & 10,03 & \multirow{2}{*}{0,283} \\
\hline Nfig_T_Pós & 10 & 43,40 & 11,15 & \\
\hline NN_T_Pré & 10 & 53,60 & 25,41 & \multirow{2}{*}{0,635} \\
\hline NN_T_Pós & 10 & 54,30 & 20,56 & \\
\hline CF_Pré & 10 & 6,80 & 0,42 & \multirow{2}{*}{0,157} \\
\hline CF_Pós & 10 & 7,00 & 0,00 & \\
\hline Matem_Pré & 10 & 6,60 & 1,78 & \multirow{2}{*}{0,180} \\
\hline Matem_Pós & 10 & 6,90 & 1,79 & \\
\hline Memor_Pré & 10 & 8,70 & 0,95 & \multirow{2}{*}{0,157} \\
\hline Memor_Pós & 10 & 8,90 & 0,99 & \\
\hline Contg_Pré & 10 & 54,60 & 13,00 & 0713 \\
\hline Contg_Pós & 10 & 54,50 & 12,09 & $0, / 13$ \\
\hline Ritmo_Pré & 10 & 3,40 & 0,70 & $>0999$ \\
\hline Ritmo_Pós & 10 & 3,40 & 0,70 & $>0,999$ \\
\hline M_I_Pré & 10 & 3,80 & 1,23 & $>0999$ \\
\hline M_I_Pós & 10 & 3,80 & 1,23 & $>0,999$ \\
\hline Seq_Pré & 10 & 2,00 & 0,00 & $>0,999$ \\
\hline Seq_Pós & 10 & 2,00 & 0,00 & $>0,995$ \\
\hline
\end{tabular}

Legenda: Alf_: alfabeto; $C_{-}$: cópia de formas; Matem_: matemática; Dit_P_: ditado de palavras; DitNP_: ditado de não-palavras; Memor_: memória; Cor1m_: palavras lidas corretamente em 1 minuto; PC_: palavras lidas corretamente; LP_: leitura de palavras; LNP_: leitura de não-palavras; Alit_: aliteração; Contg_: contagem; RepP_: repetição de palavras; RepNP_: repetição de não-palavras; Nfig_T_: nomeação de figuras; NN_T_: nomeação de números; MV2_: memória visual duas sequências; MV3_: memória visual três sequências; MV4_: memória visual quatro sequências; MV5_: memória visual cinco sequências; D_S_: discriminação de sons; M_I_: memória indireta; Seq_: sequências. 
Tabela 3 - Distribuição das médias, desvio-padrão e significância de p do desempenho dos escolares do Gle em situação de pré-testagem e pós-testagem

\begin{tabular}{|c|c|c|c|c|}
\hline Par de Variáveis & $\mathbf{n}$ & Média & Desvio-padrão & Significância (p) \\
\hline Alf_Pré & 10 & 19,10 & 2,23 & \multirow{2}{*}{$0,016^{*}$} \\
\hline Alf_Pós & 10 & 21,20 & 2,74 & \\
\hline LP_Pré & 10 & 516,50 & 322,39 & \multirow{2}{*}{$0,005^{*}$} \\
\hline LP_Pós & 10 & 265,30 & 181,45 & \\
\hline LNP_Pré & 10 & 68,20 & 83,51 & \multirow{2}{*}{$0,013^{*}$} \\
\hline LNP_Pós & 10 & 32,50 & 17,04 & \\
\hline Cor1m_Pré & 10 & 9,70 & 6,27 & \multirow{2}{*}{$0,008^{*}$} \\
\hline Cor1m_Pós & 10 & 15,60 & 8,90 & \\
\hline Dit_P_Pré & 10 & 6,70 & 3,37 & \multirow{2}{*}{$0,015^{\star}$} \\
\hline Dit_P_Pós & 10 & 8,90 & 3,21 & \\
\hline DitNP_Pré & 10 & 1,60 & 0,70 & \multirow{2}{*}{$0,010^{*}$} \\
\hline DitNP_Pós & 10 & 2,80 & 1,03 & \\
\hline Alit_Pré & 10 & 7,90 & 1,73 & \multirow{2}{*}{0,608} \\
\hline Alit_Pós & 10 & 8,10 & 1,45 & \\
\hline Rima_Pré & 10 & 13,80 & 3,82 & \multirow{2}{*}{$0,049^{*}$} \\
\hline Rima_Pós & 10 & 16,30 & 3,68 & \\
\hline D_S_Pré & 10 & 3,30 & 1,70 & \multirow{2}{*}{$0,005^{\star}$} \\
\hline D_S_Pós & 10 & 16,30 & 3,53 & \\
\hline RepP_Pré & 10 & 2,60 & 0,70 & \multirow{2}{*}{$0,004^{*}$} \\
\hline RepP_Pós & 10 & 4,90 & 0,74 & \\
\hline RepNP_Pré & 10 & 3,40 & 0,52 & \multirow{2}{*}{$0,005^{\star}$} \\
\hline RepNP_Pós & 10 & 6,30 & 0,67 & \\
\hline PC_Pré & 10 & 31,10 & 12,21 & \multirow{2}{*}{$0,007^{\star}$} \\
\hline PC_Pós & 10 & 49,10 & 15,33 & \\
\hline MV2_Pré & 10 & 2,30 & 0,67 & \multirow{2}{*}{$0,004^{*}$} \\
\hline MV2_Pós & 10 & 4,00 & 0,00 & \\
\hline MV3_Pré & 10 & 1,80 & 0,63 & \multirow{2}{*}{$0,004^{*}$} \\
\hline MV3_Pós & 10 & 4,00 & 0,00 & \\
\hline MV4_Pré & 10 & 1,40 & 0,84 & \multirow{2}{*}{$0,004^{*}$} \\
\hline MV4_Pós & 10 & 4,00 & 0,00 & \\
\hline MV5_Pré & 10 & 1,80 & 0,42 & \multirow{2}{*}{$0,003^{*}$} \\
\hline MV5_Pós & 10 & 4,00 & 0,00 & \\
\hline Nfig_T_Pré & 10 & 99,60 & 19,52 & \multirow{2}{*}{$0,005^{\star}$} \\
\hline Nfig_T_Pós & 10 & 43,60 & 4,79 & \\
\hline NN_T_Pré & 10 & 93,60 & 20,22 & \multirow{2}{*}{$0,005^{\star}$} \\
\hline NN_T_Pós & 10 & 48,80 & 10,55 & \\
\hline CF_Pré & 10 & 5,50 & 2,22 & \multirow{2}{*}{0,492} \\
\hline CF_Pós & 10 & 5,50 & 2,42 & \\
\hline Matem_Pré & 10 & 3,50 & 2,68 & \multirow{2}{*}{$0,034^{*}$} \\
\hline Matem_Pós & 10 & 4,90 & 3,84 & \\
\hline Memor_Pré & 10 & 6,90 & 2,23 & \multirow{2}{*}{$0,009^{*}$} \\
\hline Memor_Pós & 10 & 8,00 & 2,36 & \\
\hline Contg_Pré & 10 & 166,80 & 281,86 & 0093 \\
\hline Contg_Pós & 10 & 191,20 & 116,95 & 0,093 \\
\hline Ritmo_Pré & 10 & 3,30 & 0,95 & \\
\hline Ritmo_Pós & 10 & 5,60 & 1,35 & $0,004^{*}$ \\
\hline M_I_Pré & 10 & 1,70 & 0,48 & 0.004 * \\
\hline M_I_Pós & 10 & 4,20 & 0,79 & \\
\hline Seq_Pré & 10 & 0,90 & 0,32 & ค ค २०* \\
\hline Seq_Pós & 10 & 2,00 & 0,00 & 0,002 \\
\hline
\end{tabular}

Legenda: Alf_: alfabeto; $C_{-}$: cópia de formas; Matem_: matemática; Dit_P_: ditado de palavras; DitNP_: ditado de não-palavras; Memor_: memória; Cor1m_: palavras lidas corretamente em 1 minuto; PC_: palavras lidas corretamente; LP_: leitura de palavras; LNP_: leitura de não-palavras; Alit_: aliteração; Contg_: contagem; RepP_: repetição de palavras; RepNP_: repetição de não-palavras; Nfig_T_: nomeação de figuras; NN_T_: nomeação de números; MV2_: memória visual duas sequências; MV3_: memória visual três sequências; MV4_: memória visual quatro sequências; MV5_: memória visual cinco sequências; D_S_: discriminação de sons; M_I_: memória indireta; Seq_: sequências. 
Tabela 4 - Distribuição das médias, desvio-padrão e significância de p do desempenho dos escolares do Glle em situação de pré-testagem e pós-testagem

\begin{tabular}{|c|c|c|c|c|}
\hline Par de Variáveis & $\mathrm{n}$ & Média & Desvio-padrão & Significância (p) \\
\hline Alf_Pré & 10 & 24,00 & 2,21 & \multirow{2}{*}{$0,041^{*}$} \\
\hline Alf_Pós & 10 & 25,80 & 0,42 & \\
\hline LP_Pré & 10 & 231,80 & 93,03 & \multirow{2}{*}{0,093} \\
\hline LP_Pós & 10 & 189,20 & 91,90 & \\
\hline LNP_Pré & 10 & 18,90 & 5,70 & \multirow{2}{*}{$0,011^{*}$} \\
\hline LNP_Pós & 10 & 13,70 & 2,75 & \\
\hline Cor1m_Pré & 10 & 26,30 & 10,69 & \multirow{2}{*}{$0,005^{\star}$} \\
\hline Cor1m_Pós & 10 & 36,20 & 9,57 & \\
\hline Dit_P_Pré & 10 & 13,30 & 4,83 & \multirow{2}{*}{$0,005^{\star}$} \\
\hline Dit_P_Pós & 10 & 18,50 & 5,04 & \\
\hline DitNP_Pré & 10 & 3,20 & 1,32 & \multirow{2}{*}{$0,011^{*}$} \\
\hline DitNP_Pós & 10 & 5,00 & 1,15 & \\
\hline Alit_Pré & 10 & 8,90 & 0,99 & \multirow{2}{*}{$0,026^{*}$} \\
\hline Alit_Pós & 10 & 9,90 & 0,32 & \\
\hline Rima_Pré & 10 & 14,30 & 5,93 & \multirow{2}{*}{$0,018^{*}$} \\
\hline Rima_Pós & 10 & 17,80 & 2,44 & \\
\hline D_S_Pré & 10 & 17,30 & 4,16 & \multirow{2}{*}{$0,043^{*}$} \\
\hline D_S_Pós & 10 & 19,60 & 0,97 & \\
\hline RepP_Pré & 10 & 3,70 & 1,49 & \multirow{2}{*}{0,063} \\
\hline RepP_Pós & 10 & 4,30 & 0,95 & \\
\hline RepNP_Pré & 10 & 4,40 & 0,84 & \multirow{2}{*}{$0,014^{*}$} \\
\hline RepNP_Pós & 10 & 5,00 & 0,67 & \\
\hline PC_Pré & 10 & 28,00 & 10,01 & \multirow{2}{*}{$0,005^{\star}$} \\
\hline PC_Pós & 10 & 33,20 & 12,20 & \\
\hline MV2_Pré & 10 & 2,90 & 0,74 & \multirow{2}{*}{$0,009^{*}$} \\
\hline MV2_Pós & 10 & 4,00 & 0,00 & \\
\hline MV3_Pré & 10 & 3,80 & 1,03 & \multirow{2}{*}{$0,020^{*}$} \\
\hline MV3_Pós & 10 & 4,80 & 0,79 & \\
\hline MV4_Pré & 10 & 5,30 & 1,57 & \multirow{2}{*}{0,809} \\
\hline MV4_Pós & 10 & 5,40 & 0,97 & \\
\hline MV5_Pré & 10 & 6,30 & 1,70 & \multirow{2}{*}{0,131} \\
\hline MV5_Pós & 10 & 6,90 & 1,20 & \\
\hline Nfig_T_Pré & 10 & 40,10 & 7,89 & \multirow{2}{*}{$0,008^{*}$} \\
\hline Nfig_T_Pós & 10 & 32,50 & 7,68 & \\
\hline NN_T_Pré & 10 & 39,70 & 7,75 & \multirow{2}{*}{$0,016^{*}$} \\
\hline NN_T_Pós & 10 & 33,70 & 6,20 & \\
\hline CF_Pré & 10 & 6,60 & 0,52 & \multirow{2}{*}{$0,046^{*}$} \\
\hline CF_Pós & 10 & 7,00 & 0,00 & \\
\hline Matem_Pré & 10 & 4,20 & 1,62 & \multirow{2}{*}{$0,004^{*}$} \\
\hline Matem_Pós & 10 & 6,70 & 1,34 & \\
\hline Memor_Pré & 10 & 8,20 & 1,23 & \multirow{2}{*}{$0,004^{*}$} \\
\hline Memor_Pós & 10 & 11,00 & 1,05 & \\
\hline Contg_Pré & 10 & 89,70 & 85,72 & 0838 \\
\hline Contg_Pós & 10 & 79,00 & 15,26 & 0,838 \\
\hline Ritmo_Pré & 10 & 2,60 & 1,07 & \\
\hline Ritmo_Pós & 10 & 5,20 & 1,23 & $0,004^{\star}$ \\
\hline M_I_Pré & 10 & 3,20 & 0,79 & $0031^{*}$ \\
\hline M_I_Pós & 10 & 4,20 & 1,40 & $0,031^{x}$ \\
\hline Seq_Pré & 10 & 2,00 & 0,00 & \\
\hline Seq_Pós & 10 & 2,00 & 0,00 & $>0,999$ \\
\hline
\end{tabular}

Legenda: Alf_: alfabeto; $C_{-}$: cópia de formas; Matem_: matemática; Dit_P_: ditado de palavras; DitNP_: ditado de não-palavras; Memor_: memória; Cor1m_: palavras lidas corretamente em 1 minuto; PC_: palavras lidas corretamente; LP_: leitura de palavras; LNP_: leitura de não-palavras; Alit_: aliteração; Contg_: contagem; RepP_: repetição de palavras; RepNP_: repetição de não-palavras; Nfig_T_: nomeação de figuras; NN_T_: nomeação de números; MV2_: memória visual duas sequências; MV3_: memória visual três sequências; MV4_: memória visual quatro sequências; MV5_: memória visual cinco sequências; D_S_: discriminação de sons; M_I_: memória indireta; Seq_: sequências. 
Tabela 5 - Distribuição das médias, desvio-padrão e significância de $\mathrm{p}$ do desempenho dos escolares do GI e GIl em situação de pré-testagem e pós-testagem para leitura e compreensão do texto lido

\begin{tabular}{|c|c|c|c|c|}
\hline & Par de Variáveis & Média & Desvio-padrão & Significância (p) \\
\hline \multirow{4}{*}{$\frac{\omega}{\sigma}$} & TT_Pré & 990,60 & 546,12 & \multirow{2}{*}{$0,002^{*}$} \\
\hline & TT_Pós & 598,30 & 289,53 & \\
\hline & Comp_Pré & 30,00 & 15,39 & \multirow{2}{*}{$0,026^{*}$} \\
\hline & Comp_Pós & 46,25 & 36,52 & \\
\hline \multirow{4}{*}{$\frac{0}{0}$} & TT_Pré & 271,95 & 108,29 & \multirow{2}{*}{0,654} \\
\hline & TT_Pós & 276,85 & 130,79 & \\
\hline & Comp_Pré & 43,75 & 32,32 & \multirow{2}{*}{$0,046^{*}$} \\
\hline & Comp_Pós & 53,75 & 38,28 & \\
\hline \multirow{4}{*}{$\frac{\varpi}{\bar{\sigma}}$} & TT_Pré & 493,70 & 157,11 & \multirow{2}{*}{$0,007^{*}$} \\
\hline & TT_Pós & 369,70 & 127,46 & \\
\hline & Comp_Pré & 40,00 & 21,08 & \multirow{2}{*}{$0,016^{*}$} \\
\hline & Comp_Pós & 67,50 & 16,87 & \\
\hline \multirow{4}{*}{$\frac{\amalg}{\bar{\sigma}}$} & TT_Pré & 359,40 & 67,85 & \multirow{2}{*}{0,169} \\
\hline & TT_Pós & 382,40 & 90,44 & \\
\hline & Comp_Pré & 27,50 & 7,91 & \multirow{2}{*}{0,180} \\
\hline & Comp_Pós & 35,00 & 17,48 & \\
\hline
\end{tabular}

Legenda: TT_: tempo total ; Comp_: compreensão.

\section{DISCUSSÃO}

Neste estudo, pôde-se verificar similaridade de respostas entre os grupos I e II, o que possibilita pensar em um perfil de habilidades cognitivo-linguísticas tanto para os escolares com dislexia como para os escolares sem histórico de dificuldades de aprendizagem.

Entretanto, depois da realização do programa de remediação fonológica, pôde-se observar que os escolares, tanto do Gl como do Gll, apresentaram melhores desempenhos.

Entre as habilidades cognitivo-linguísticas, verificou-se que a leitura de palavras, leitura de pseudopalavras, repetição de pseudopalavras, memória para dígitos, aliteração e contagem de número em ordem inversa foram os testes que apresentaram melhora depois da aplicação do programa de remediação fonológica.

A eficácia do programa de remediação fonológica também foi evidenciada na leitura e compreensão do texto lido, pois os escolares do Gle e do Glle apresentaram um desempenho melhor depois da realização do programa, ocorrendo diminuição do número de palavras lidas por minuto, diminuição do tempo total de leitura e compreensão do texto lido. Entretanto, apesar de não ter sido submetido ao programa de remediação fonológica, o Glc apresentou melhora na compreensão do texto lido.
Os escolares disléxicos deste estudo apresentaram melhora nas habilidades cognitivo-linguísticas com a realização do programa, devido ao programa ter desenvolvido habilidades de discriminação. Trata-se de habilidade que, por estar comprometida em disléxicos, gera alterações em leitura e escrita de palavras e pseudopalavras ${ }^{20,21}$.

Da mesma forma, os escolares com dislexia e os escolares sem histórico de dificuldades de aprendizagem apresentaram melhora nas habilidades cognitivo-linguísticas, apontando para a necessidade de uma reflexão sobre a metodologia de alfabetização utilizada em situação de sala de aula, pois as habilidades de atenção, discriminação e percepção dos sons trabalhados no programa proporcionaram aumento na sensibilidade fonológica, o que se refletiu também na melhora do tempo e compreensão da leitura.

Em situação de pós-testagem, pôde-se observar que ambos os grupos submetidos ao programa melhoram o seu desempenho em subtestes de memória de trabalho, como repetição de nãopalavras, nomeação automática rápida e memória para dígitos. Atualmente ${ }^{22,23}$, sabe-se que a memória fonológica de curta duração ou loop fonológico não é muito eficiente nos escolares com dislexia, porém, pode-se verificar nos dados deste estudo que os escolares que não apresentam problemas de aprendizagem se beneficiaram com a instrução 
do programa quanto à memória de trabalho, o que gerou impacto sobre a compreensão da leitura desses escolares.

Os resultados deste estudo evidenciaram também que os escolares com dislexia apresentaram dificuldade quanto à leitura de palavra e pseudopalavras, leitura rápida de palavras e pseudopalavras, discriminação de sons, nomeação automática rápida, consciência fonológica e memória verbal ${ }^{24-26 .}$

Em situação de pós-testagem, pode-se verificar que os escolares disléxicos apresentaram melhor desempenho na maioria das habilidades cognitivo-linguísticas avaliadas, mostrando que estes se beneficiam de um tratamento que explore as habilidades do processamento linguístico, pois, os escolares disléxicos apresentam disfunções em mecanismos de percepção responsáveis pelo processamento auditivo temporal da informação. Consequentemente, o problema da percepção da fala causa um efeito em cascata, iniciando com o rompimento do desenvolvimento normal do sistema fonológico e resultando em problemas na aprendizagem da leitura e da soletração ${ }^{27}$.

Esse efeito também foi observado nos escolares sem histórico de dificuldades de aprendizagem, evidenciando que as habilidades trabalhadas no programa devem ser inseridas no contexto de sala se aula, pois tanto os escolares disléxicos como os demais escolares poderiam ser beneficiados de um ensino voltado para a percepção fonológica do sistema de escrita do português brasileiro.

Assim, os escolares disléxicos, no contexto de sala de aula, poderiam superar a dificuldade com a memória verbal, melhorando o desempenho na decodificação e compreensão da leitura e aumentando o vocabulário ${ }^{26,28}$.

Neste estudo, verificou-se que tanto os escolares com dislexia como os escolares sem histórico de problemas de aprendizagem melhoraram seu desempenho em estratégias de percepção, discriminação, armazenamento e recuperação de informação silábica e fonêmica, conforme descrito em estudos internacionais ${ }^{7,29}$ e estudos nacionais ${ }^{12-14}$.
Entretanto, há um aspecto deste estudo importante a ser considerado: os escolares disléxicos que não foram submetidos ao programa também melhoram quanto à compreensão do texto lido, evidenciando que o papel da escola foi fundamental para a ocorrência deste fenômeno. Contudo, o mesmo não ocorreu com o tempo de leitura, o que demonstra uma falta de concordância entre o trabalho em sala de aula no que se refere à fluência e compreensão de leitura. Isso também foi evidenciado em estudo descrito na literatura ${ }^{30}$.

Os achados deste estudo, no que se refere aos escolares sem dificuldades de aprendizagem, foram os mesmos encontrados na literatura ${ }^{15,31}$, o que evidencia que o uso de estratégias fonológicas associadas à leitura é eficaz para o desenvolvimento e melhora das condições de leitura, demonstrando que a instrução fonológica deve ser enfocada para a aprendizagem da leitura e escrita ${ }^{32-35}$.

\section{CONCLUSÃO}

Conclui-se que os escolares com dislexia do desenvolvimento apresentaram desempenho inferior em relação aos escolares sem dificuldades de aprendizagem nas habilidades cognitivo-linguísticas em situação de pré-testagem.

A realização do programa de remediação fonológica foi eficaz tanto para os escolares disléxicos como para os escolares sem dificuldades de aprendizagem, devido à melhora das habilidades de leitura de palavras, pseudopalavras, memória de trabalho e nomeação rápida evidenciada nas duas populações de escolares.

A melhora dos escolares com dislexia do desenvolvimento e sem dificuldades de aprendizagem, submetidos ao programa de remediação fonológica em situação de pós-testagem, evidencia a necessidade de a instrução fonológica ser oferecida no contexto da alfabetização, pois isso auxiliaria os escolares a desenvolverem habilidades metalinguísticas para a aprendizagem da base alfabética do sistema de escrita do português brasileiro. 


\begin{abstract}
Purposes: to compare the findings of pre-test and post-test evaluation in students with developmental dyslexia and good readers submitted to phonological remediation program and to check the therapeutic effectiveness of phonological remediation program in students with developmental dyslexia. Methods: 40 students of the $2^{\text {nd }}$ to $4^{\text {th }}$ grades of public schools of Marília-SP, both genders, from to 8 to 12-year old took part in this study; distributed in Gl: 20 students with the interdisciplinary diagnosis of developmental dyslexia and Gll: 20 good readers paired according to gender, age and school level. All students were submitted to the Cognitive-Linguistic Performance Test in the collective and individual version, oral reading and text comprehension. Results: the results showed statistically significant differences indicating that the GI and Gll students submitted to phonological remediation program showed higher performance in post-test when compared to pre-test in the most evaluated cognitive-linguistic skills, including reading and text comprehension. Conclusion: the phonological remediation program was effective for students with or without developmental dyslexia, suggesting that the sound-letter relation skill should be used in the classroom context in order to improve the reading skills for these students.
\end{abstract}

KEYWORDS: Dyslexia; Reading; Intervention Studies

\section{REFERÊNCIAS}

1. Silani G, Frith U, Demonet JF, Fazio F, Perni D, Price $C$, et al. Brain abnormalities underlying altered activation in dyslexia: a voxel based morphometry study. Brain. 2005; 128(10):2453-61.

2. Galaburda AM. Dyslexia-- a molecular disorder of neuronal migration: the 2004 Norman Geschwind Memorial Lecture. Annals Dyslexia. 2005; 55(2):151-65.

3. Joanisse MF, Manis FR, Keating P, Seidenberg MS. Language deficits in dyslexic children: speech perception, phonology, and morphology. J Exp Child Psychol. 2000; 77(1):30-60.

4. Broom YM, Doctor EA. Developmental phonological dyslexia: a case study of the efficacy of a remediation programme. Cognit Neuropsychol. 1995; 12(7):725-66.

5. Gonzalez MRO, Espinel AIG, Rosquete RG. Remedial interventions for children with reading disabilities: speech perception--an effective component in phonological training? J Learn Disabil. 2002; 35(4):334-42.

6. Calhoon MB. Effects of a peer-mediated phonological skill and reading comprehension program on reading skill acquisition for middle school students with reading disabilities. J Learn Disabil. 2005; 38(5):424-33.

7. Simos PG, Sarkari S, Billingsley RL, Fletcher JM, Denton C, Papanicolaou AC. Altering the brain circuits for reading through intervention: a magnetic source imaging study. Neuropsychol. 2007; 21(4):485-6.
8. Braga SML. Remediação da leitura: um estudo com crianças de primeiro grau utilizando a técnica de cloze. [dissertação] São Paulo (SP): Universidade de São Paulo; 1981.

9. Graminha SSV, Machado VLS, Francischini EL, Befi VM. Emprego de um procedimento de treino gradual de discriminação de sílabas em crianças com dificuldades na leitura e escrita. Arq Bras Psicol. 1987; 39(1):84-94.

10. Santos AAA. A influência da consciência fonológica na aquisição da leitura e escrita. In: Sisto FF, organizador. Atuação psicopedagógica e aprendizagem escolar. Rio de Petrópolis: Vozes. 1996; p. 213-47.

11. Capovilla AGS, Capovilla FC. Efeitos do treino de consciência fonológica em crianças com baixo nível sócio-econômico. Psicol Reflex Crit. 2000; 13(1):7-24.

12. Capellini SA, Ciasca SM. Avaliação da consciência fonológica em crianças com distúrbio específico de leitura e escrita e distúrbio de aprendizagem. Temas Desenvolv. 2000; 8(48):17-23.

13. Capellini SA, Padula NAMR, Ciasca SM. Desempenho de escolares com distúrbio específico de leitura em programa de remediação. Pró-Fono. 2004; 16(3):261-74.

14. Salgado CA, Capellini SA. Programa de remediação fonológica em escolares com dislexia do desenvolvimento. Pró-Fono. 2008; 20(1):31-6.

15. Hatcher PJ, Hulme C, Ellis AW. Ameliorating early reading failure by integrating the teaching of reading 
and phonological skills: the phonological linkage hypothesis. Child Develop. 1994; 65(1):41-57.

16. Smythe IS, Everatt J. International dyslexia test (IDT). University of Surrey: University; 2000.

17. Capellini SA, Silva C, Gonzaga J, Tegeiro MG, Villa PC, Smythe I. Desempenho cognitivolinguístico de escolares de 1a a 4a séries do ensino público municipal. Psicopedagogia. Ass Bras Psicopedag. 2007; 24:30-44.

18. Condemarin M, Blomquist M. Dislexia: manual de leitura corretiva. Porto Alegre: Artes Médicas; 1989. p. 51-8.

19. Ramos CS. Avaliação da leitura em escolares com indicação de dificuldade de leitura e escrita. [dissertação] São Paulo (SP): Universidade Federal de São Paulo; 2005.

20. Kujala T, Lovio R, Lepisto $T$, Laansonen M, Naatanen R. Evaluation of multi-attribute auditory discrimination in dyslexia with the mismatch negativity. Clin Neurophysiol. 2006; 117(4):885-93. 21. Dufor O, Serniclaes W, Sprenger-Charolles L, Démonet JF. Top-down processes during auditory phoneme categorization in dyslexia: a PET study. Neuroimage. 2007; 34(4):1692-707.

22. Thomson JM, Richardson U, Goswami U. Phonological similarity neighborhoods and children's short-term memory: typical development and dyslexia. Memory Cognition. 2005; 33(7):1210-9.

23. Serrano F, Defior S. Dyslexia speed problems in a transparent orthography. Ann Dyslexia. 2008; 58(1):81-95.

24. Paul I, Bott C, Wienbruch C, Elbert TR. Word processing differences between dyslexic and control children. BMC Psychiatry [periodic online]. 2006; 6:5. Available from: URL: http://www.biomedcentral. com/1471-244X/6/5.

25. Bonte ML, Poelmans H, Blomert L. Deviant neurophysiological responses to phonological regularities in speech in dyslexic children. Neuropsychologia. 2007; 45(7):1427-37.
26. Puolakanaho A, Ahonen T, Aro M, Eklund K, Leppänen PH, Poikkeus AM, et al. Developmental links of very early phonological and language skills to second grade reading outcomes. J Learn Disabil. 2008; 41(4):353-70.

27. Boets $B$, Wounters $J$, van Wieringen $A$, Ghesquiére P. Auditory processing, speech perception and phonological ability in pre-school children at high-risk for dyslexia: a longitudinal study of the auditory temporal processing theory. Neuropsychologia. 2007; 45(8):1608-20.

28. Banai K, Ahissar M. Auditory processing deficits in dyslexia: task or stimulus related? Cereb Cortex. 2006; 16(12):1718-28.

29. Joffe V, Pring T. Children with phonological problems: a survey of clinical practice. Int $\mathrm{J}$ Lang Commun Disord. 2008; 43(2):154-64.

30. Alves LM. A prosódia na leitura da criança disléxica. [tese] Belo Horizonte (MG): Universidade Federal de Minas Gerais; 2007. $400 \mathrm{f}$.

31. O'Shaughnessy TE, Swanson HL. A comparison of two reading interventions for children with reading disabilities. J Learn Disabil. 2000; 33(3):257-77.

32. Russo NM, Nicol TG, Zecker SG, Hayes EA, Kraus $\mathrm{N}$. Auditory training improves neural timing in the human brainstem. Behav Brain Res. 2005; 156(1):95-103.

33. Magnan A, Ecalle J. Audio-visual training in children with reading disabilities. Comput Educ. 2006; 46(4):407-25.

34. Regtvoort AGFM, van der Leij A. Early intervention with children of dyslexic parents: effects of computer-based reading instruction at home on literacy acquisition. Learn Individ Differ. 2007; 17(1):35-53.

35. Keilmann A, Wintermeyer $M$. Is a specialised training of phonological awareness indicated in every preschool child?. Folia Phoniatr Logopaed. 2008; 60(2):73-9.

DOI: 10.1590/S1516-18462009005000060

RECEBIDO EM: 23/09/2008

ACEITO EM: 14/09/2009

Endereço para correspondência:

Simone Aparecida Cappellini

Rua Bartolomeu de Gusmão, 10-84

Bauru - SP

CEP: 17017-336

E-mail: sacap@uol.com.br 